\title{
28 Nach dem Krieg: Die Krim zwischen 1856 und 1905
}

\begin{abstract}
Nachts las ich Tschechow in dem karg eingerichteten Pensionszimmer, und wenn ich morgens vom Balkon nach Westen schaute, dachte ich mir Tschechow nicht anders als mit den riesigen Felsen auf den Schultern, unter ihrer Last Worte über Ebenen aufs Papier keuchend. Ich war mit einer spärlich markierten Karte im Kopf hierher gekommen, auf der neben Tschechow der unvermeidliche Puschkin verzeichnet war, dazu Mickiewicz' Krim-Sonette, Marina Zwetajewa in Feodossja (sic), Mandelstamm in Koktebel, stets befasst mit dem Bogen von den Weißen Nächten des kalten Nordens zu diesem blauen, warmen Land, das unzerreißbare Fäden zur Antike bereithielt. An diesen Fäden ließ sich Russland vor die Wiege der Kultur spannen, an Hellas anbinden, der Bauer im nördlichen Schnee zähmen. Mehr Licht! ${ }^{1}$
\end{abstract}

Die Reiseerzählung des Autorenpaars Esther Kinsky ( $\left.{ }^{1} 1956\right)$ und Martin Chalmers (1948-2014) basiert auf einer im Oktober 2013 - also noch vor der Annexion 2014 durchgeführten Fahrt über die Halbinsel. Geschildert wird darin kein mythisiertes, schönes Zauberland, sondern eine kaum verklärte Destination, wie bereits der Untertitel „Aufzeichnungen von der kalten Krim“ andeutet. In der zitierten Passage sind einige Elemente angeführt, welche für die Wahrnehmungen der Krim nach 1856 typisch sind. Der Slavistin und Übersetzerin Kinsky sind diese KrimBilder bekannt, ist sie doch sicher mit mehr als „einer spärlich markierten Karte im Kopf" auf die Krim gereist. Treffend wird die Halbinsel als ein Ort bezeichnet, der russische und nichtrussische Autorinnen und Autoren des 19. und 20. Jahrhunderts (wie beispielsweise den genannten Polen Mickiewicz) inspirierte. Alsdann wird auf den Stolz russischer Elitenangehöriger hingewiesen, mit der Krim ,ihren' Teil an der hellenischen Hochkultur zu besitzen (Kapitel 23), was auch in der zweiten Hälfte des 19. Jahrhunderts nachwirkte. In den Jahrzehnten vor dem Ersten Weltkrieg war die Krim - und auch darauf verweisen Kinsky und Chalmers - zu einem nachgesuchten südlichen Gegenentwurf der „Weißen Nächte des kalten Nordens" geworden. Dies galt nicht nur für Künstler und Künstlerinnen, die ihren Beitrag zur Entstehung des sog. Krim-Texts, wie es in der Literaturwissenschaft heißt, und zur Mythisierung (Kapitel 2) der Halbinsel als Ort russischer Kultur geleistet haben. Dass auch eine ökonomisch und gesellschaftlich gutgestellte Hautevolee in ihren Sommerhäusern und angemieteten Refugien an der malerischen, mediterranen Südküste eine enge, auch emotionale Verbindung zur Krim aufbaute, nimmt kaum Wunder. Wie ließ sich aber der von Kinsky und Chalmers erwähnte „Bauer im nördlichen Schnee“ durch den russischen Besitz der Krim „zähmen“, also von einem vermeintlich wilden in einen kultivierten

1 Kinsky u. Chalmers (2015), 81.

Ә OpenAccess. (c) 2020 Kerstin S. Jobst, publiziert von De Gruyter. (cc))BY Dieses Werk ist lizenziert unter der Creative Commons Attribution 4.0 International. https://doi.org/10.1515/9783110520620-030 
Zustand transformieren? Und gibt es weitere ,reale Aussagen` über die Halbinsel hinter der literarisch-künstlerischen Form, der sich die AutorInnen bedienen?

Dass von der Verbindung mit der Krim ein Modernisierungsschub in das von westeuropäischen AutorInnen vielfach als „rückständig“ bezeichnete Russländische Imperium ausgegangen sei, ${ }^{2}$ drücken Kinsky und Chalmers mittels der Johann Wolfgang von Goethe zugesprochenen, aber vermutlich so nicht gefallenen berühmten letzten Worte „mehr Licht“ aus, steht Licht doch im Allgemeinen für Aufklärung und Fortschritt. Dies kann auf das ambitionierte Reformprogramm des Imperiums durch Alexander II. hinweisen, das bereits genannt worden ist (Kapitel 26) und nach dem Krimkrieg von den Verantwortlichen in Angriff genommen wurde. Dessen Kernstück war die Aufhebung der Leibeigenschaft 1861. ${ }^{3}$ Auf der Krim mit ihrer vergleichsweise niedrigen Anzahl unfreier Bauern stellte diese Maßnahme allerdings keine große Zäsur dar. Die Reformen im Bildungswesen (1863 und 1871), der Justiz (1864) und die Einführung lokaler Selbstverwaltungseinheiten (1864 und 1870) beeinflussten hingegen die Verhältnisse auf der Krim nachhaltig, wurden die Partizipationsmöglichkeiten vieler Krim-BewohnerInnen doch unabhängig von ihrer ethnischen oder religiösen Zugehörigkeit erweitert. ${ }^{4}$ Auch wenn die Schäden durch Krieg und Besatzung auf der Halbinsel erst mit Verzögerung beseitigt werden konnten, wurden mittelfristig einige grundlegende Verbesserungen erreicht. ${ }^{5}$ Dies galt vor allen Dingen für überfällige infrastrukturelle Maßnahmen: Das Straßennetz auf der Krim selbst als auch die Verbindungen nach Norden waren nach einer ersten Phase unmittelbar nach der Annexion von 1783 nämlich nicht ausreichend ausgebaut worden. Die Anbindung an das gesamtrussische Eisenbahnnetz, deren Fehlen im Krimkrieg dramatische Folgen für den russischen Nachschub gezeigt hatte, erfolgte endlich im Jahr 1875; bereits 1851 war der Anschluss genehmigt, aber nicht umgesetzt worden, was die Säumigkeit der Verantwortlichen zeigt. ${ }^{6}$

Die Anbindung an das Streckennetz ermöglichte nicht nur eine Belebung des Warenflusses, etwa den Transport der auf der Krim angebauten Agrarprodukte wie Obst, Gemüse, Wein oder Tabak in den Norden. Sie beförderte auch die Entwicklung des Tourismus, konnten mittels Eisenbahn doch viel mehr Menschen einfacher und schneller auf die Krim reisen als vordem - und sich so persönlich von deren Schönheit und Geschichtsträchtigkeit überzeugen. Dies wiederum beförderte die kollektive Verbindung vieler UntertanInnen des Zaren mit ihr, und

2 Vgl. dazu neben Wolff (1994) auch Jobst (2013b).

3 Moon (2001).

4 Vgl. zu den Folgen auf dem Gebiet der Rechtsprechung Kirmse (2013).

5 Vgl. Jobst (2007b), 403-406.

6 Baumgart (1972), 122. 
zwar ähnlich stark wie die Erinnerung an den dort ausgetragenen ,heldenhaften', aber leider vergeblichen Kampf gegen Engländer, Franzosen und Osmanen. Im Übrigen stellt dies keine russische Besonderheit dar, hat die Forschung doch gezeigt, dass der moderne Tourismus kollektive Aneignungsprozesse von Räumen und Territorien befördert. Regionen, die bis dahin unbekannt waren, werden als Teil des eigenen Staates empfunden und emotional angeeignet. ${ }^{7}$ Eine persönliche Inaugenscheinnahme entlegener touristischer Gebiete ist dazu gar nicht immer notwendig und war im Fall der Krim ohnehin für die meisten nicht leistbar. Das Wissen um ihren besonderen Wert für das Imperium wurde durch Kanäle wie Reiseberichte $^{8}$, Erzählungen oder Postkarten von Bekannten, die dort gewesen waren, bzw. durch die sich gegen Ende des 19. Jahrhunderts im Zarenreich dynamisch entwickelnden Medien weithin popularisiert. ${ }^{9}$ Der zitierte „Bauer im nördlichen Schnee“ konnte somit auch von der Besonderheit der Krim Kenntnis erlangen, selbst wenn er niemals einen Fuß dorthin setzen würde; ob ihn dies aber „, zähmte“, sei dahingestellt.

Schon in zarischer Zeit war die Krim zu einer bevorzugten Touristendestination des Imperiums geworden, deren Kapitale Jalta wurde. ${ }^{10}$ Die Metamorphose des einstmals kleinen tatarischen Dorfes war beeindruckend: Jalta konnte es hinsichtlich der BesucherInnenzahlen Ende des 19. Jahrhunderts mit dem böhmischen Karlsbad aufnehmen. ${ }^{11}$ Die wirtschaftlichen Erwartungen, die an die Krim als Ganzes gestellt wurden, sollten sich indes nicht erfüllen. ${ }^{12}$ Besonders spürbar wurde die Krise des Agrarsektors nach dem tatarischen Massenexodus infolge des Krimkriegs. Es sollte sich nämlich zeigen, dass die seit Potemkins Zeiten gehegte Phantasie einer ,besseren Krim ohne Krimtataren' Nachteile barg. Die als Ersatz für die muslimischen Bauern auf die Halbinsel verbrachten bulgarischen oder deutschen NeusiedlerInnen in den von den TatarInnen verlassenen Gebieten hatten Schwierigkeiten, das Land zu bewirtschaften. ${ }^{13}$ Der Fremdenverkehr, vor allen Dingen im südlichen Küstenort Jalta, nahm hingegen - wie auch der Weinanbau - ab der zweiten Hälfte des 19. Jahrhunderts eine gute Entwicklung. Der dortige Tourismus trug zur - durchaus nicht als ungebrochene Erfolgsgeschichte zu verstehenden, sondern ambivalente Folgen zeitigenden -

7 Vgl. Jaworski (2014), 19.

$8 \mathrm{Zu}$ den zahlreichen Reiseberichten über die Krim in russischer Sprache vgl. Nepomniaščij (1999).

9 Telesko (2014).

10 Dazu im Überblick Usyskin (2000); McReynolds (2003).

11 Mal'gin (2006), 100.

$12 \mathrm{Zu}$ den ersten Jahrzehnten unter russischer Herrschaft vgl. Lynch (1965).

13 Vgl. den zeitgenössischen Bericht Kratkij otčet (1868). 
Modernisierung und zur Entwicklung der Konsumgesellschaft in diesem Teil des Russländischen Reiches bei. ${ }^{14}$ Die Verwaltung Jaltas und anderer Küstengebiete stand durch den Reiseboom vor großen Aufgaben, musste doch die gesamte Infrastruktur - Straßen, die Versorgung mit Wasser und Energie und so weiter optimiert werden.

Begonnen hatte die Entwicklung der Halbinsel zu einem nachgesuchten Ziel mit der sog. Taurischen Reise der Zarin Katharina II. 1787 (Kapitel 23). Darauf folgten die gelehrten Touristen wie Peter Simon Pallas, dem Männer und Frauen aus ganz Europa folgten. ${ }^{15}$ Deren Berichte wurden gerne gelesen, erfreute sich das Genre des Reiseberichtes doch in der ersten Hälfte des 19. Jahrhunderts großer Beliebtheit. Dies galt umso mehr für Krim-Beschreibungen, war diese doch bereits in der Antike bedeutend gewesen und landschaftlich schön. Im Zusammenhang mit der sog. Orientalischen Frage und der militärischen Relevanz der Krim zog es schließlich auch Reisende wie den Engländer Laurence Oliphant (1829-1888) dorthin: Sein auf der Grundlage einer im Herbst 1852 durchgeführten Krim-Reise ein Jahr später veröffentlichter und immens erfolgreicher Bericht kam zur rechten Zeit, zumal er sowohl als Unterhaltungsliteratur als auch als eine Art Spionagereport gelesen wurde. Dieser gab zugleich Auskunft über die militärische Schlagkraft der auf der Halbinsel stationierten zarischen Armee und Marine. ${ }^{16}$ Nach dem Krimkrieg zog es dann allerlei Schlachtfeldtouristen auf die Halbinsel, die ebenfalls einen erheblichen Anteil an der Popularisierung des Reiseziels hatten. Eine noch größere Rolle spielte dabei allerdings die kaiserliche Familie der Romanovs selbst.

Im Jahre 1825 hatte diese mit dem Oreanda-Palast ein erstes Anwesen in der Nähe Jaltas erworben, welches allerdings vorerst nur sporadisch genutzt wurde. Erst mit der Herrschaft Alexanders II. verbrachte die Zarenfamilie regelmäßig Urlaube auf der Halbinsel. In dem ehemals verschlafenen Küstenort wurde also schon von dem Februar 1945 und der berühmten Jalta-Konferenz (Kapitel 35) Politik gemacht: Nach dem Krimkrieg entstanden zahlreiche repräsentative Bauten nebst einer erforderlichen ,hauptstädtischen` Infrastruktur, da Jalta und Umgebung in den Sommermonaten zum temporären imperialen Regierungssitz wurden. Der tatkräftig in Angriff genommene Ausbau des Telegraphen- und Straßennetzes beispielsweise resultierte nicht zuletzt aus der Begeisterung der Romanovs für die Halbinsel. ${ }^{17}$ Und diese Begeisterung übertrug sich auf Adlige

14 Dazu Böröcz (1992).

15 Deutsche und Franzosen stellten nach Russen die wohl größte Zahl, aber auch Polen wie K. Kaczkowski bereisten die Halbinsel und hinterließen Berichte: Kaczkowski (1829).

16 Oliphant (1854). Vgl. auch dessen Biographie bei Taylor (1982).

17 N. Kalinin u. Zemljaničenko (1993), 122-124. 
und finanziell potente Angehörige höherer Schichten. Ende des 19. Jahrhunderts ,entdeckten' auch die Mittelschichten - und auch diese gab es in einem gewissen Ausmaß im Russländischen Reich - die Krim für sich.

Als Lenin (Vladimir I. Ul’janov, 1870-1924) im Dezember 1920 in seinem berühmten Dekret „Über den Gebrauch der Krim für die medizinische Behandlung der Werktätigen“ („Ob ispol'zovanij Kryma dlja lečenija trudjaščichsja“) ${ }^{18}$ die Rolle der frisch und diesmal dauerhaft von den Bol'ševiki eingenommenen Halbinsel als wichtigen Kurort Sowjetrusslands bzw. der Sowjetunion (ab 1922) definierte, konnte er auf bereits in vorrevolutionärer Zeit gelegte Grundlagen zurückgreifen. Die vom Aufbau des Sozialismus erschöpften ProletarierInnen erholten sich fortan in den enteigneten und umgebauten Villen und Palästen des Adels und der Bourgeoisie; neue Sanatorien kamen hinzu.

Auch wenn ein Massentourismus nach unseren heutigen Maßstäben erst in sowjetischer Zeit einsetze und die Krim damit zur ,Badewanne der UdSSR` werden sollte, so hatte sich die Südküste bereits in zarischer Zeit irreversibel verändert. Die Moderne mit all ihren Widersprüchlichkeiten zog ein: Einerseits brachten der Tourismus und das Kurwesen positive Dinge mit sich, neben den ausgebauten Straßen vergrößerte sich zum Beispiel das Sortiment an dargebotenen Waren, und die ärztliche Versorgung in Jalta und Umgebung verbesserte sich. ${ }^{19}$ Die Architektur veränderte sich, und die damals entstandenen prachtvollen Villen und andere Bauwerke ziehen bis in unsere Zeit Reisende an und begeistern sie. Andererseits beklagten schon im 19. Jahrhundert nicht wenige KrimbewohnerInnen und Auswärtige die hohen Lebenshaltungskosten und den Verlust des exotischen Flairs. ${ }^{20}$ Viele Einheimische profitierten aber von diesem neuen Wirtschaftszweig und nahmen das Geschäft mit den Zugereisten beherzt in ihre Hände: Mit der Einführung der Selbstverwaltungsorgane - der schon erwähnten zemstva wurden der Tourismussektor und das Kurwesen ab 1871 eine Angelegenheit örtlicher Akteure. Ein Pionier und Exponent der im Kapitalismus sehr üblichen Verquickung privater und politischer Interessen wurde z. B. Jaltas erster gewählter Bürgermeister Sergej P. Galachov (1806-1873), dem zugleich das beste Hotel vor Ort gehörte. ${ }^{21}$ Nicht nur slavische KrimbewohnerInnen machten Geschäfte mit den Reisenden, dies taten auch die anderen nationalen Gruppen: Krimtataren etwa arbeiteten als Kutscher, Eisverkäufer oder Bergführer. ${ }^{22}$ Der Tourismus und

18 Vgl. den Text unter Dekret SNK (1920).

19 Fisher (1978), 99.

20 Dazu Jobst (2007b), 340.

21 Mal'gin (2006), 95-96.

22 Mal'gin (2006), 105. 
das Kurwesen veränderte also das Leben der KrimbewohnerInnen unabhängig ihrer Nationalität.

Dass im 19. Jahrhundert „das Kurhaus als nationale Aufgabe“ zu betrachten ist, wurde von der Forschung an vielen Beispielen exemplifiziert. ${ }^{23}$ Und dies gilt auch für Russland und die Krim, brandmarkten doch manche russische AutorInnen des 19. Jahrhunderts die Vorliebe reicher RussInnen für ausländische Spas wie Baden-Baden oder Bad Ems als unpatriotisch und empfahlen stattdessen eine Erholungsreise in die heimischen Kurbäder - in den Kaukasus, in das Baltikum oder aber in das „russische Italien“, also auf die Krim. ${ }^{24}$ Letztere wurde zunehmend nicht mehr als fremdes, muslimisches Gebiet empfunden oder gar als Kolonie, sondern als integraler Teil des eigenen Vaterlandes, was der Reiseschriftsteller und Ethnograph Evgenij Markov (1835-1903) auf den Punkt brachte und damit vielen Landsleuten aus der Seele sprach: „Alles, was Du suchst, findest Du in Deinem eigenen Land, an der Südküste der Krim.“25

23 Fuhs (1992), 173.

24 Mazurevskij (1845), hier 27.

25 Markov (1994), 245. 